\title{
An Improved Teaching-Learning-Based Optimization Algorithm for Sphericity Error Evaluation
}

\author{
YangYang $^{\text {a) }}$, LiMing ${ }^{\text {b) }}$, Gu JingJun ${ }^{\text {c) }}$ \\ a)mryyfi@i.shu.edu.cn \\ b)robotlib@shu.edu.cn \\ c)sdulmhsh@163.com
}

School of Mechatronic Engineering and Automation, Shanghai University, Shanghai 200072, China

\begin{abstract}
In order to improve the accuracy and the convergence speed of the sphericity error, an improved teaching and learning algorithm is proposed to evaluate the sphericity error. Based on the basic teaching-learning-based optimization, the initial solution quality is improved by logistic chaotic initialization; At the end of each iteration, the interpolation algorithm is applied to the global optimal solution to further improve the search accuracy of the algorithm. Finally, one group of sphericity error algorithm though the measurement data in the related literature is verified the effectiveness of the ITLBO, the test result show that the ITLBO algorithm has advantages in the calculating accuracy and iteration convergence speed, and it is very suitable for the application in the sphericity error evaluation.
\end{abstract}

Keywords: Teaching-Learning-Based, Optimization Algorithm, Sphericity Error Evaluation

\section{INTRODUCTION}

For the spherical error, there is no relevant standard to unify its error assessment methods at this stage, but as other common form error, the evaluation method can be used for reference in the form error such as roundness, cylindricity error evaluation. Among them, the minimum zone method is one of the methods which has a higher evaluation accuracy. It can meet the minimum zone principle in the international standards and obtain the accurate results of the measured data.

In recent years, the minimum zone method of sphericity error has been studied as follows: Samuel applied the method of computational geometry to the solution of sphericity error [1], Cui calculated the sphericity error by genetic algorithm [2], Wen used the immune evolutionary algorithm to the evaluation of sphericity error [3], Hu applied the improved particle swarm optimization method to assess the sphericity error accurately [4], Luo used artificial bee colony algorithm to solve the sphericity error of the measured parts[5], Lei respectively used mesh search algorithm [6] and the geometric approximation algorithm [7] to evaluate the accuracy of sphericity error, Liu constructed the geometrical model of spherical error by the method of chord truncation, and it can obtain the spherical error accurately [8]. In the above sphericity error evaluation algorithm, the intelligent 
optimization algorithm such as genetic algorithm and particle swarm algorithm, is widely used because of its simple mathematical model. But on the other hand, the intelligent optimization algorithms have the strong parameter dependence, which is slow in the late iteration and easy to get into the local optimal, therefore, it needs to further improve the performance in the algorithm.

Teaching-learning-based algorithm is a new intelligent optimization algorithm, which requires few parameters and has relatively simple computational principle, and it is very suitable for engineering applications $[9,10]$. In order to improve the calculation accuracy and convergence speed of sphericity error, the teaching-learning-based algorithm is applied to the evaluation of sphericity error. But for the basic teaching-learning-based algorithm, there are some problems such as the premature convergence in iteration process. Therefore, two improved strategies are designed in this paper to improve the searching capability of TLBO algorithm and accelerate its convergence speed. And the effectiveness of the algorithm is verified by experiments.

\section{MATHEMATICAL MODEL OF SPHERICITY ERROR EVALUATION}

As shown in Figure 1, $f$ is the measured area which is contained by concentric sphere $O$ (the solid line) and $O$ '(the dashed line), when the $f$ takes the minimum value, the value is equal to the sphericity error of the measured data. Therefore, when the measured sphere data points have been obtained, how to find the concentric sphere coordinates which satisfies the sphericity error $f$ under the minimum zone method has become the key problem.

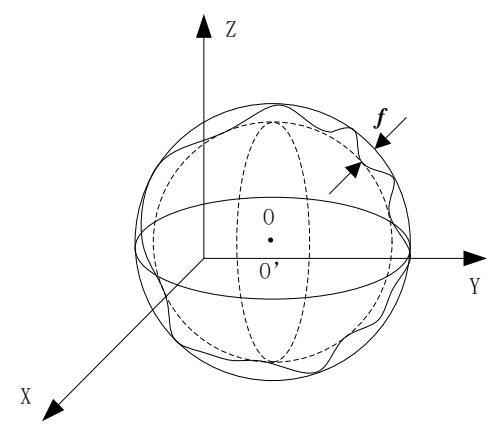

FIGURE 1. Schematic of the spherical error

For space-point set $P_{i}\left(x_{i}, y_{i}, z_{i}\right)(i=1,2, \ldots, n)$, the space distance between the any point measured to the sphere center $O\left(x_{o}, y_{o}, z_{o}\right)$ is shown in formula (1), where $r_{i}$ is the distance between the measured point to the sphere. According to the theory of spherical error, the essence of the problem is a minimization optimization problem. Therefore, the set of points objective function under the minimum zone principle can be established as shown in formula (2), where $\max \left(r_{i}\right)$ is the maximum sphere radius of the concentric sphere containing the point set, and $\min \left(r_{i}\right)$ is the smallest radius, $f$ is the spherical error value, so the problem can be converted to find the value of the parameters $x_{o}, y_{o}, z_{o}$ which can meet the minimum zone condition of $f$.

$$
\begin{aligned}
& r_{i}=\sqrt{\left(x_{i}-x_{0}\right)^{2}+\left(y_{i}-y_{0}\right)^{2}+\left(z_{i}-z_{0}\right)^{2}} \quad i=1,2, \ldots, n \\
& \quad f\left(x_{o}, y_{o}, z_{o}\right)=\min \left(\max \left(r_{i}\right)-\min \left(r_{i}\right)\right)
\end{aligned}
$$




\section{IMPROVED TEACHING-LEARNING-BASED OPTIMIZATION}

For the basic teaching-learning-based optimization algorithm[9], with the iterative process, the population diversity will be premature loss, making the solution fall into local optimization easily. Therefore, in order to increase the diversity of the population and prevent the premature convergence of the algorithm, two methods are introduced into the TLBO.

\section{Chaos Initialization}

In order to distribute the initial population more evenly and improve the quality of the initial solution of the algorithm, the chaotic random number is introduced into the basic TLBO. Chaos has the characteristics of traversal, randomness and regularity, and it is obtained through a deterministic equation[11]. The initial solution sequence generated by logistic chaotic[12] mapping is presented as formula (3) and (4).

$$
\begin{gathered}
x_{n+1}=\mu x_{n}\left(1-x_{n}\right) \\
x_{n}^{\prime}=x_{\min }+x_{n}\left(x_{\max }-x_{\min }\right)
\end{gathered}
$$

Where $\mu$ is the control parameter, when $\mu$ is 4 , the system is a completely chaotic; $x_{\min }, x_{\max }$ are the upper and lower bounds of the variable respectively; $x_{n}$ is a chaotic initialization variable in $[-1,0)$ and $(0,1] ; x_{n}^{\prime}$ is the variable which is mapped to the range of value $x_{\min }$ between $x_{\max }$ by $x_{n}$.

\section{Quadratic interpolation}

The interpolation algorithm[13] is applied to the TLBO global optimal individual, and the new individual location is generated near the optimal solution, compared to the two fitness function values between the two solution, then update the optimal solution, and further enhance the searching capability of the algorithm. The quadratic interpolation algorithm is shown in formula(5).

$$
x_{n e w, i}=\frac{\left(x_{t, i}^{2}-x_{b, i}^{2}\right) f\left(x_{a}\right)+\left(x_{b, i}^{2}-x_{a, i}^{2}\right) f\left(x_{t}\right)+\left(x_{a, i}^{2}-x_{t, i}^{2}\right) f\left(x_{b}\right)}{2\left[\left(x_{t, i}-x_{b, i}\right) f\left(x_{a}\right)+\left(x_{b, i}-x_{a, i}\right) f\left(x_{t}\right)+\left(x_{a, i}-x_{t, i}\right) f\left(x_{b}\right)\right]}
$$

Where $x_{t}$ is the global optimal solution; $x_{a}$ and $x_{b}$ are the randomly selected solutions respectively; $x_{n e w, i}$ is a new solution which is created by $x_{t}, x_{a}$ and $x_{b}$.

\section{Algorithm Steps of ITLBO}

Based on the corresponding improvement methods, the proposed ITLBO algorithm process is shown below:

(1) Parameters initialization . $N$ is the population number; $D$ is the dimension of problem; $x_{\min }$ and $x_{\max }$ are the upper and lower limit of the variables. Then go to step (2).

(2) Chaos Initialization. A random sequence individuals which number is $N$ are generated, and the fitness function value of each individual is calculated; The teacher $x_{t}$ in class is the individual who has the minimum value. Then go to step (3).

(3) Teaching stage. The students' scores are updated according to the the teaching stage. Then go to step (4).

(4) Learning stage. The students learn from each other. And record global optimal solution $x_{t}$. Go to step (5).

(5) Interpolation algorithm. The best solution $x_{t}$ is updated by interpolation algorithm, seen as formula (5) . Then go to step (6).

(6) Judgement of iteration stopping criterion. If the calculation satisfies the stopping criterion, if it is satisfied, the calculation is completed and the optimal solution is output; if not, return step (3). 


\section{TEST ANALYSIS}

\section{Sphericity Error Experiment}

In this experiment, the sphericity error is carried out by using the second group data of literature[14], the data is shown in table 3.The calculation results of table 3are shown in table 4. The calculation results of TLBO and other related literature algorithms are list in table 4 . It can be seen that the calculation results of sphericity error of TLBO and ITLBO is smallest, so the accuracy of the two algorithms has a higher accuracy than other algorithms such as least square method. The calculation results of ITLBO and TLBO are $0.009662 \mathrm{~mm}$ and the concentric sphere coordinates is $(0.0035096,-0.003305,-0.00029195)$, their results are the smallest in the listed algorithms, so the accuracy of the sphericity error is highest. The iterative curve of TLBO and ITLBO is shown in figure 5. Although the iterative results of the two algorithms are same, the convergence speed and accuracy of ITLBO are better than those of TLBO. The ITLBO converges in 18 times, and the TLBO algorithm converges in 25 times.

TABLE 1. Spherical error of data [14]

\begin{tabular}{ccccc}
\hline Method & $\boldsymbol{x}_{\mathbf{0}}$ & $\boldsymbol{y}_{\mathbf{0}}$ & $\boldsymbol{z}_{\mathbf{0}}$ & error \\
\hline Least-squares method[14] & - & - & & -0.0132 \\
MZM $_{4-1 \text { Model }}[14]$ & -0.003224 & -0.003484 & 0.000349 & 0.01259 \\
MZM $_{\text {1-4Model }}[14]$ & 0.004816 & -0.002009 & -0.000424 & 0.00993 \\
MZM $_{\text {3-2Model }}[14]$ & -0.000018 & -0.003241 & 0.000275 & 0.01002 \\
MZM $_{2-3 \text { Model }}[14]$ & 0.003509 & -0.003305 & -0.000292 & 0.00967 \\
IEA[3] & 0.003506 & -0.003308 & -0.000292 & 0.009669 \\
IGA[3] & - & - & - & 0.009678 \\
EGA[3] & - & - & - & 0.056038 \\
TLBO & 0.0035096 & -0.003305 & -0.00029195 & 0.009662 \\
ITLBO & 0.0035096 & -0.003305 & -0.00029195 & 0.009662 \\
\hline
\end{tabular}



FIGURE 2. Sphericity error iteration curve of TLBO and ITLBO algorithms 


\section{CONCLUSION}

In order to improve the accuracy and convergence speed of the spherical error, the teaching-learning-based algorithm is applied to the evaluation of the spherical error. An improved teaching and learning algorithm is proposed to solve the problems of early premature convergence of basic teaching-learning-based algorithm and easy to get into local optimization. In order to improve the quality of the initial solution of the algorithm, chaos Initialization is designed to initialize the population; Finally, in order to prevent the algorithm from getting into the local optimal later, the interpolation algorithm is introduced into the late algorithm, and the algorithm is verified by experiments. The results shows that the improved teaching-learning-based algorithm is very suitable for the accurate evaluation of the spherical error.

\section{ACKNOWLEDGEMENT}

This paper is supported by national high technology research and development program of china(No. 2015AA043003) .

\section{REFERENCES}

[1] G.L. Samuel, M.S. Shunmugam. Evaluation of sphericity error from form data using computational geometric techniques, Int. J. Mach. Tool. Manu. 42.3 (2002):405-416.

[2] C.C. Cui, R.S. Che, Y.E. Dong, et al. Sphericity error evaluation using the genetic algorithm, Opt. Precision. Eng. 10.4 (2002):333-339.

[3] X.L. Wen. An immune evolutionary algorithm for sphericity error evaluation, Int. J. Mach. Tool. Manu. 44.10 (2004):1077-1084.

[4] J. Hu, C.C. Cui, F.G. Huang. Sphericity error evaluation based on a modified particle swarm optimizer using dynamic inertia weight, J. Graph. 33.5 (2012):99-103.

[5] J. Luo, H. Wu, Q. Wang. Sphericity error evaluation with Double-quick Artificial Bee colony algorithm, Acta Metrologica Sinica. 32.6 (2011):501-504.

[6] X.Q. Lei, H.W. Song, Y. J. Xue, et al. Sphericity Error Based on Mesh Search Algorithm, Trans Chin Soc Agr Mach. 43.5 (2012):222-225.

[7] X.Q. Lei, Z. Gao, M. Duan, et al. Method for sphericity error evaluation using geometry optimization searching algorithm, Precis Eng. 42.5 (2015):101-112.

[8] F. Liu, G.H. Xu, Liang L et al. Intersecting Chords Method in Minimum Zone Evaluation of Sphericity Deviation, J. mech eng. 52.5 (2016):137-143.

[9] R.V. Rao, V. J. Savsani, D. P. Vakharia. Teaching-learning-based optimization: A novel method for constrained mechanical design optimization problems, Comput Aided Design. 43.3 (2011):303-315.

[10] C.V. Camp, M. Farshchin. Design of space trusses using modified teaching-learning based optimization, Eng Struct, (2014):87-97.

[11] B. Li, W. S. Jiang. Chaos optimization method and its application, Control Theory A.14.4 (1997):613-615.

[12] A. Kanso, N. Smaoui. Logistic chaotic maps for binary numbers generations, Chaos Soliton Fract. 40.5 (2009):2557-2568.

[13] M. M. Ali, A. Törn, S. Viitanen. A Numerical Comparison of Some Modified Controlled Random Search Algorithms, 
J Global Optim. 11.4 (1997):377-385.

[14] C.K. Chen, C.H. Liu. A study on analyzing the problem of the spherical form error, Precis Eng. 24.2 (2000):119-126 